\title{
Evidence for a unidirectional retrograde communication between distal His bundle and atria ${ }^{\star}$
}

\author{
JAY W MASON \\ From the Cardiology Division, Stanford University School of Medicine, Stanford, California, USA
}

SUMMARY Intracardiac electrogram recordings are presented suggesting the existence in one patient with recurrent supraventricular tachycardia of a functionally unidirectional retrograde connection between the distal His bundle and the low right atrium. Recordings obtained during junctional rhythm showed a short ventriculoatrial conduction time which did not lengthen during rapid or premature ventricular pacing. During an episode of supraventricular tachycardia induced by a single atrial premature stimulus, an infra-His Wenckebach period occurred during which retrograde ventriculoatrial conduction remained constant until ventricular capture was blocked, but atrial activation persisted with the same retrograde atrial activation sequence seen in the previous beats of supraventricular tachycardia. The findings appear to document participation in supraventricular tachycardia of a type of atrio-His fibre originally described by Brechenmacher.

In 1974 and 1975 Brechenmacher presented histological evidence for the existence of atrial fibres bypassing the human atrioventricular node and terminating directly in the His bundle. ${ }^{12} \mathrm{He}$ found such direct atrio-His connections in only two of a series of 687 hearts studied histologically. He specifically differentiated the fibres from so-called 'James fibres', which pass from the atrium to the lower atrioventricular node or to the base of the tricuspid valve, and which both he and James consider to be present normally in most, if not all, human hearts. ${ }^{2}{ }^{3}$ The purpose of this paper is to present unusual electrophysiological findings in a single patient which are best explained by an atrio-His fibre of Brechenmacher functioning retrogradely only.

\section{Case report}

The patient was a 53-year-old woman who complained of a recurrent sensation of rapid heart action since adolescence which had recently worsened despite medical treatment. Twenty-four hours of ambulatory monitoring showed only two brief runs of supraventricular tachycardia with rates around 170 beats per minute. The resting surface electrocardiogram was within normal limits. The PR interval was $0 \cdot 16 \mathrm{~s}$ and there was no delta wave.

\footnotetext{
^This work was supported in part by an NIH grant.
}

Electrophysiological study was undertaken to identify the mechanism of the arrhythmia and to identify an antiarrhythmic regimen which could prevent its recurrence.

\section{Methods}

The patient underwent intracardiac electrophysiological study. Quadripolar electrode catheters (interpolar distance $=1 \mathrm{~cm}$ ) were advanced to the right atrium and the bundle of $\mathrm{His}$ position and a hexipolar electrode catheter was positioned at the right ventricular apex and later repositioned in the coronary sinus. Standard recording and stimulating studies were performed, including description of anterograde and retrograde conduction and refractoriness by atrial and ventricular rapid pacing and programmed extrastimulation. Extrastimuli were delivered singly and in pairs during sinus rhythm and at multiple drive rates to the right atrium, coronary sinus, and right ventricle to induce supraventricular tachycardia. Unfortunately, no induced episodes were sufficiently prolonged to permit assessment of the effect of premature atrial or ventricular stimulation upon the tachycardia.

INTERPRETATION OF ELECTROPHYSIOLOGICAL RECORDINGS

Recordings during spontaneous sinus rhythm were normal. The atrial and right ventricular effective 
refractory periods, and anterograde atrioventricular nodal refractory periods were normal. The anterograde atrioventricular nodal refractory curve was smooth, without evidence for dual conduction pathways. Wenckebach atrioventricular nodal conduction block appeared at an atrial pacing cycle length of $380 \mathrm{~ms}$. Ventricular pre-excitation could not be demonstrated during pacing at multiple rates from high right atrium, low right atrium, or coronary sinus. During right ventricular pacing, the retrograde atrial activation sequence was low-tohigh right atrium, and the right ventricular to low right atrial conduction time remained short and almost constant (90 to $105 \mathrm{~ms}$ ) at all ventricular pacing rates to a maximum of 150 beats per minute (Fig. 1). In addition, during right ventricular extrastimulation (minimum V1 V2 achieved $=300 \mathrm{~ms}$ ) the retrograde conduction time increased by only $15 \mathrm{~ms}$. It is conceivable that greater retrograde conduction delay might have been found if the ventricle had been paced at rates greater than 150 beats per minute, or if right ventricular extrastimulation had been performed at rapid ventricular drive rates. Supraventricular tachycardia was easily and repeatedly induced with single or pairs of premature right atrial and coronary sinus extrastimuli. These tachycardias often followed induced single sinus node re-entrant beats (Fig. 2 and 3 ). The cycle length of supraventricular tachycardia was $330 \mathrm{~ms}$. The atrial activation sequence was reversed (low right atrium to high right atrium). The coronary sinus electrogram followed the low and preceded the high right atrial signal. The $\mathrm{AH}$ interval during tachycardia was long, the QRS morphology unaltered, and the HV interval remained constant during most episodes.

The data reported so far suggest the presence of a concealed retrograde bypass short-circuiting the atrioventricular node. As judged from the retrograde atrial activation sequence, the atrial end of the fibre is right-sided. The origin of the ventricular end of the fibre cannot be determined precisely from the data presented so far, though the distal connection must be at or below the end of the atrioventricular node to explain the constancy of ventriculoatrial conduction time. Spontaneous episodes of infra-Hisian Wenckebach block during reentry help us to localise the distal insertion of this fibre (Fig. 2 and 3). In the episode illustrated in Fig. 2, a premature right atrial stimulus leads to a sinuatrial nodal re-entry beat, after which re-entrant supraventricular tachycardia with reversed atrial activation sequence ensues (beats 4 to 6 in Fig. 2). During the tachycardia infra-Hisian Wenckebach block occurs. The HV interval associated with QRS complex beat 2 is normal $(45 \mathrm{~ms})$. The HV interval of the premature beat 3 is increased to $90 \mathrm{~ms}$. On subsequent beat 4 , the HV interval becomes normal after a pause caused by atrioventricular nodal conduction delay. The HV interval is increased again with the next cycle (beat 5) and complete block below the His occurs between beats 5 and 6 . The HV interval is normal again on the sixth and seventh beats. The most important findings here are that: (1) ventriculoatrial conduction time remains constant despite $\mathrm{HV}$ interval a
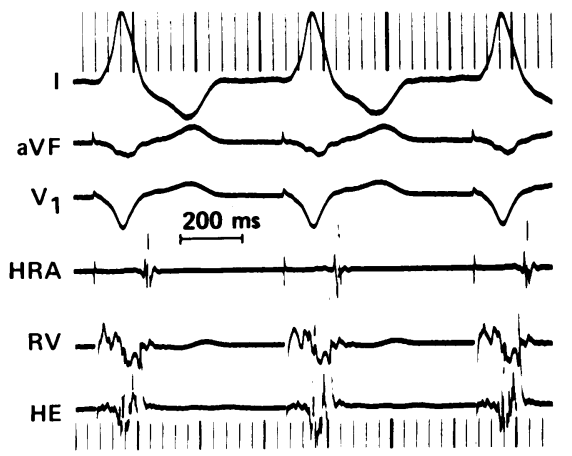

b
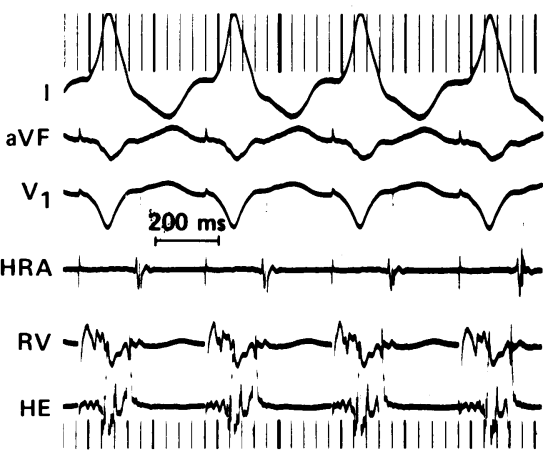
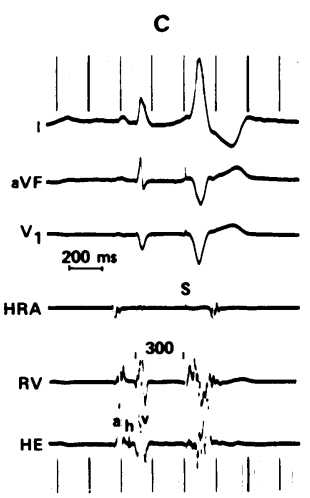

Fig. 1 Surface electrocardiographic leads $I, a V F$, and $V 1$ are recorded with three intracardiac electrograms. The boldest vertical time lines indicate one second intervals, and the lines of lesser boldness correspond to 200 and $40 \mathrm{~ms}$ intervals. The high right atrium ( $H R A$ ), right ventricular inflow ( $R V)$, and His bundle (HE) electrograms are shown recorded at paper speeds of 50 and $100 \mathrm{~mm} / \mathrm{s}$. In (a) right ventricular apical pacing at a cycle length of $600 \mathrm{~ms}$ results in a short ventriculoatrial conducticn time $(90 \mathrm{~ms})$ measured from the beginning of the right ventricular electrogram to the beginning of the low atrial electrogram in HE. With a decrease in pacing cycle length to $400 \mathrm{~ms}$ (b) there is a slight increase in ventriculoatrial conduction time to $100 \mathrm{~ms}$. In (c) the maximally premature ventricular extrastimulus (V1V2 $=300 \mathrm{~ms}$ ) fails to prolong ventriculoatrial conduction time. 
prolongation while HA time varies; and (2) retrograde atrial activation remains intact despite infraHisian block. The former finding rules against the possibility of a James bypass fibre inserting in the distal atrioventricular node or the atrioventricular nodal-His bundle junction, since a constant retrograde conduction time between $\mathrm{His}$ and atrium would have been recorded. The second finding eliminates the possibility of a septal Kent fibre, since ventricular activation was not required between beats 5 and 6 to maintain the ventriculoatrial limb of the re-entry circuit. In the ladder diagram (Fig. 2 and 3) in which an upper His bundle (UH) and a lower His bundle (LH) are depicted for illustrative purposes, the upper His bundle is assumed to be the region responsible for generation of the His spike, and the lower His bundle the site of infra-Hisian conduction delay and eventual block. It is in this lower region of the His bundle, just before the branching portion of the HisPurkinje system, where the bypass fibre's distal end seems to communicate with the conduction system. For maintenance of a constant ventriculoatrial time, the distal insertion of the bypass fibre in the bundle of His must be beyond the site of $\mathrm{HV}$ conduction delay, but above the site of conduction block.

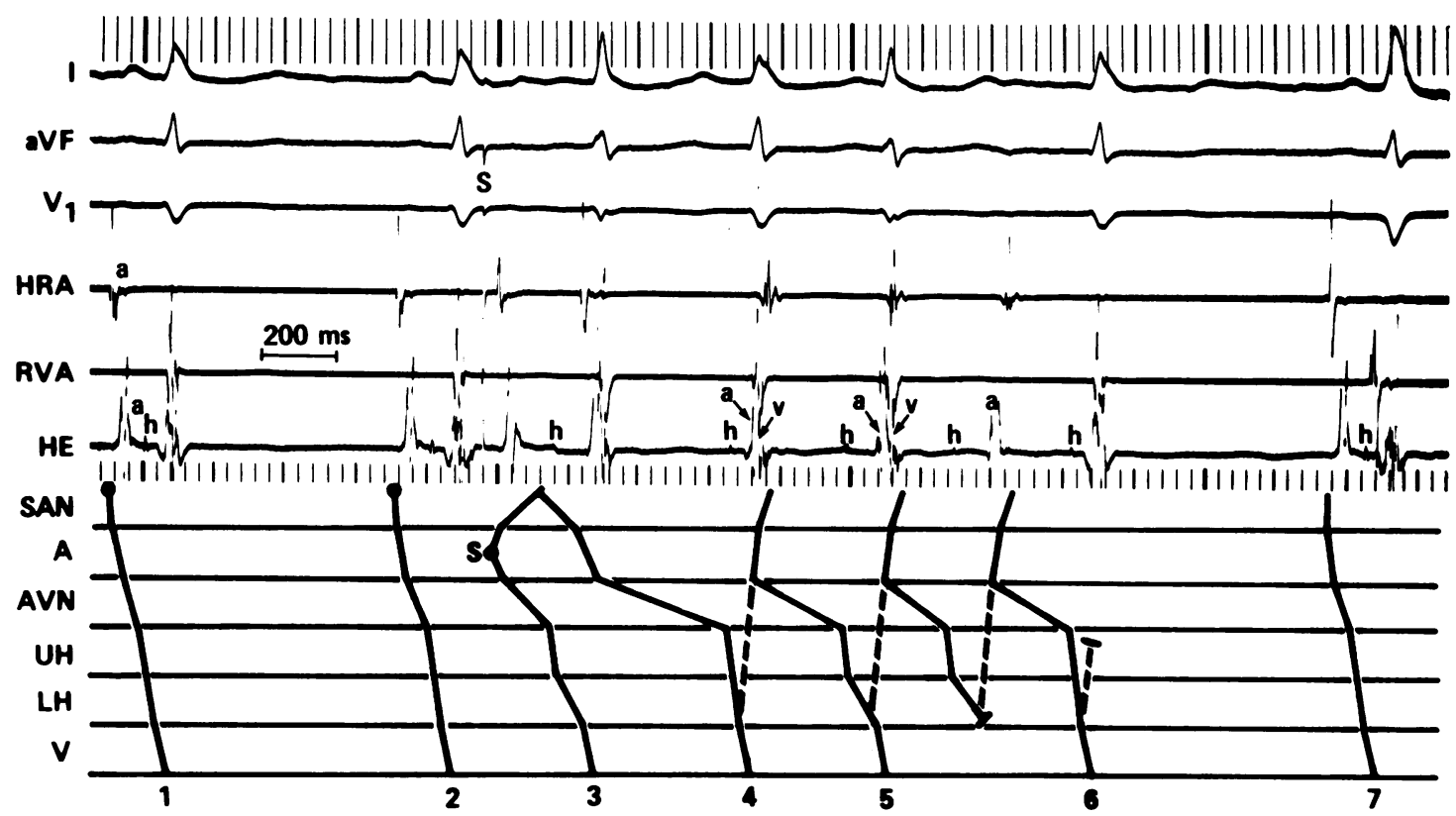

Fig. 2 This illustration supplies evidence for the location of the input end of the concealed ventriculoatrial bypass fibre. The same surface and.intracardiac leads shown in the previous illustrations are displayed, with a ladder diagram below the electrophysiological recordings. In the ladder diagram: $S A N$, sinuatrial node; $A$, atrium; $A V N$, atrioventricular node; UH, upper His bundle; LH, lower His bundle; $V$, ventricle. The first and second ventricular beats are of sinus origin. A single premature stimulus delivered to the high right atrium produces prolongation of both the AH and $H V$ intervals and results in ventricular complex 3, via anterograde conduction. An atrial echo beat follows, presumably as a result of sinuatrial nodal re-entry, suggested by maintenance of the same high to low atrial activation sequence and atrial electrogram morphology seen in the previous sinus beats. With this atrial repetition pronounced lengthening of the AH interval occurs, $H V$ interval prolongation disappears, and QRS 4 results. Another atrial repetition immediately follows the onset of ventricular complex 4, with low to high atrial activation. This represents the first of a three-beat run of re-entrant supraventricular tachycardia using the proposed atrio-His bypass. The second beat (QRS 5) of this supraventricular tachycardia is identical to the first beat, except that there is HV interval prolongation (from 45 to $90 \mathrm{~ms}$ ). Note that the $45 \mathrm{~ms}$ prolongation of $\mathrm{HV}$ interval with this beat was not associated with any change in ventriculoatrial timing. A His electrogram is recorded after beat 5 , but no ventricular electrogram or QRS follows. However, the third atrial beat of the three-beat run occurs despite the infra-His block. It should be noted further that the HA conduction time is inconstant during all three beats of supraventricular tachycardia. $Q R S$ complex 6 is the last one generated by the run of supraventricular tachycardia. Tachycardia is interrupted because of failure of retrograde atrial capture after QRS 6. Thus, these recordings show a run of supraventricular tachycardia with retrograde atrial activation, infra-His Wenckebach block, inconstant $H A$ conduction time, and constant ventriculoatrial conduction time, with preservation of atrial activation despite failure of ventricular capture. Mild terminal QRS aberrancy occurs in beats 3 and 5, both of which show HV interval prolongation. 
Fig. 3 is provided to show the presence of a critical $\mathrm{AH}$ delay as a requirement for induction of supraventricular tachycardia in this patient. This lends support to the hypothesis that a mechanism of re-entry is responsible for the arrhythmia.

One additional explanation for these findings should be considered. It is conceivable that the site of re-entry actually existed in the atrioventricular node, and that constancy of ventriculoatrial conduction times and inconstancy of HA conduction times were accounted for by, coincidentally, exactly opposite changes in retrograde atrioventricular nodal and anterograde HV times from beat to beat. This seems an unlikely explanation, because it requires an improbable coincidence. The presence of fixed ventriculoatrial conduction time during ventricular pacing strongly favours a nonAV nodal pathway serving as the retrograde limb of the re-entry circuit.

If we conclude that the ventricular end of the bypass fibre cannot terminate in the atrioventricular node or the proximal $\mathrm{His}$ bundle, one other possibility must be considered: distal termination on the right or left proximal bundle-branch. Such a fibre could account for all of the electrophysiological findings presented. But, in order for
A

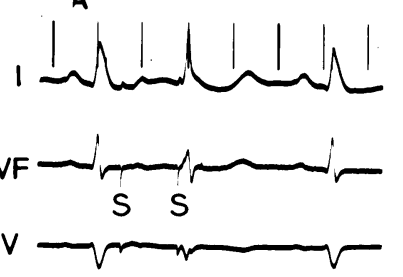

$|290| 260$

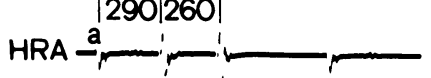

B
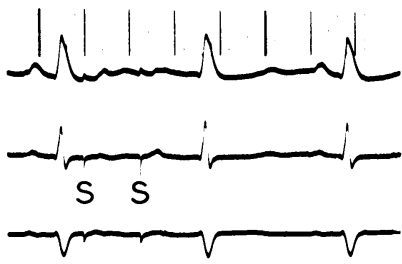

|280|260|

a

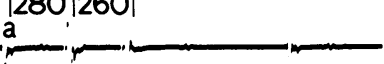

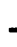

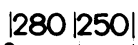

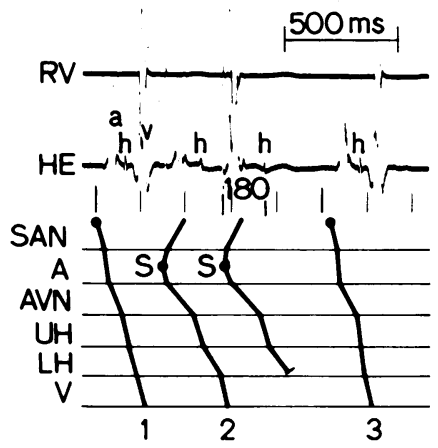
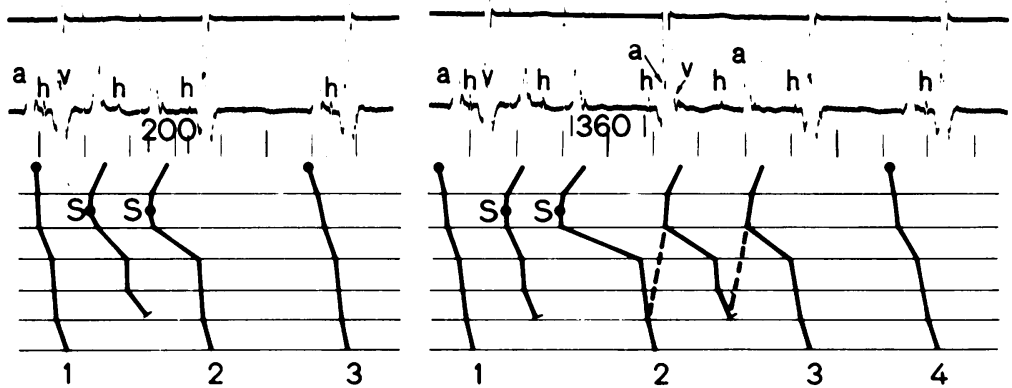

Fig. 3 The illustration shows that a critical atrioventricular noda lconduction delay is required for the proposed re-entrant phenomenon to occur. In each of the three panels, surface electrocardiographic leads $I$, aVF, and V1 are displayed with the high right atrial, right ventricular, and His bundle electrograms, and explanatory ladder diagrams appear below each panel. Abbreviations are the same as those used in Fig. 2. In each of the three illustrations, a pair of atrial extrastimuli is delivered during sinus rhythm. In (A) the first extrastimulus (labelled S) is delivered 290 ms after the last spontaneous atrial complex and results in minimal AH prolongation and considerable $H V$ prolongation from 45 to $90 \mathrm{~ms}$. The second extrastimulus, also labelled S, occurs $260 \mathrm{~ms}$ after the previous one and results in further AH prolongation to $180 \mathrm{~ms}$, followed by infra-Hisian conduction block.

In (B) the first stimulus is moved $10 \mathrm{~ms}$ closer, which results in a further small increase in AH interval, and infraHisian conduction block. The second extrastimulus remains $260 \mathrm{~ms}$ after the first, and the resultant AH interval is increased to $200 \mathrm{~ms}$. His ventricular conduction is intact, but no re-entry is seen.

In (C) the first extrastimulus is kept $280 \mathrm{~ms}$ after the last spontaneous beat and again results in infra-Hisian conduction block. The second extrastimulus is moved $10 \mathrm{~ms}$ closer to the first stimulus, resulting in an increase in AH conduction time to $360 \mathrm{~ms}$. This degree of atrioventricular nodal conduction delay results in re-entry and retrograde atrial activation. When this first atrial echo (after $Q R S$ complex 2) re-enters the conduction system anterogradely, infra-Hisian block again occurs, but the re-entry phenomenon then terminates with retrograde block. With the same premature stimulus intervals, AH delays between 200 and $360 \mathrm{~ms}$ were achieved without appearance of re-entry. Thus, a critical degree of $A V$ nodal conduction delay was required for the echo phenomena, supporting the previous conclusion that re-entry is involved. 
such a fibre to be re-entered during the episode of infra-Hisian Wenckebach block (Fig. 2 and 3), one would have to require that the anterogradely propagated impulse went beyond the bifurcation point of the proximal bundle-branch system, entered the retrograde bypass fibre, and then was blocked in both distal bundle-branch systems before activation of any ventricular myocardium.

\section{Discussion}

Convincing electrophysiological evidence for concealed atrioventricular bypass pathways-that is, channels bypassing portions of the atrioventricular conduction system and capable of conducting only in the retrograde, ventriculoatrial direction-has been presented in the past four years. ${ }^{4-7}$ In 1974, Narula pointed out that unidirectional retrograde atrioventricular nodal bypass circuits were present in a surprisingly high proportion (at least $15 \%$, and, perhaps, as much as $47 \%$ ) of patients with demonstrable ventriculoatrial conduction. ${ }^{4}$ More recently, Sung ${ }^{7}$ and Gillette ${ }^{6}$ and their collaborators have established that many patients with re-entrant supraventricular tachycardia utilised concealed retrograde atrioventricular bypasses for initiation and maintenance of their re-entrant supraventricular arrhythmias.

While the absence of typical atrioventricular nodal delay during ventriculoatrial conduction suggests a functional bypass of the atrioventricular node, the anatomical substrait for this physiological finding is uncertain. The atrial fibres described by James, ${ }^{3}$ which circumvent portions or all of the atrioventricular node to terminate at the atrioventricular nodal-His bundle junction or at the base of the tricuspid valve, might possibly function as unidirectional ventriculoatrial communications, with rapid, non-decremental conduction. A physiologicalpathological correlation has not been performed, and would be nearly impossible to achieve. In two reports, Brechenmacher described atrioventricular bypass fibres coursing through septal tissue to connect the atria with the bundle of His. ${ }^{12} \mathrm{He}$ pointed out that direct atrio-His connections could be differentiated from the fibres of James by virtue of their direct communication with the bundle of His rather than the distal atrioventricular node or the nodal-His junction. Brechenmacher considered these atrio-His bridges to be relatively rare, and could present only circumstantial evidence for a functional significance of the fibres.

On superficial examination of the recordings herein, several explanations for the data might seem tenable. Among these possibilities are: (1) a James fibre terminating at the lower portion of the atrioventricular node; (2) a James fibre terminating at the atrioventricular node-His bundle junction; (3) a septal Kent fibre; and (4) a typical atrioventricular nodal re-entrant phenomenon. Arguments against these possible explanations have been offered. Electrophysiological findings in this report suggest unidirectional retrograde ventriculo-atrial bypass physiology best explained by the presence of an atrio-His bypass fibre similar to the second case of Brechenmacher ${ }^{2}$ in which the fibre inserts into the distal His bundle just above its branching point.

\section{References}

${ }^{1}$ Brechenmacher C, Laham J, Iris L, Gerbaux A, Lenegre J. Etude histologique des voies anormale de conduction dans un syndrom de Wolff-Parkinson-White et dans un syndrome de Lown-Ganong-Levine. Arch Mal Coeur 1974; 67: 507-19.

${ }^{2}$ Brechenmacher C. Atrio-His bundle tracts. Br Heart $\mathcal{F}$ 1975; 37: 853-5.

${ }^{3}$ James TN. Morphology of the human atrioventricular node, with remarks pertinent to its electrophysiology. Am Heart f 1961 ; 62: 756-71.

${ }^{4}$ Narula OS. Retrograde pre-excitation. Comparison of antegrade and retrograde conduction intervals in man. Circulation 1974; 50: 1129-43.

${ }^{5}$ Spurrell RAJ, Krikler DM, Sowton E. Concealed bypasses of the atrioventricular node in patients with paroxysmal supraventricular tachycardia revealed by intracardiac electrical stimulation and verapamil. $A m \mathcal{F}$ Cardiol 1974; 33: 590-5.

${ }^{6}$ Gillette PC. Concealed anomalous cardiac conduction pathways: a frequent cause of supraventricular tachycardia. Am f Cardiol 1977; 40: 848-52.

'Sung RJ, Gelband H, Castellanos A, Aranda JM, Myerburg RJ. Clinical and electrophysiologic observations in patients with concealed accessory atrioventricular bypass tracts. Am $\mathcal{F}$ Cardiol 1977; 40: 839-47.

Requests for reprints to Dr Jay W Mason, Stanford University Medical Center, Stanford, California 94305, USA. 